\title{
Mechanisms of the Impact of Hashimoto Thyroiditis on Papillary Thyroid Carcinoma Progression: Relationship with the Tumor Immune Microenvironment
}

\author{
Oksana Sulaieva $^{1}$, Olena Chernenko ${ }^{2}$, Oleksiy Selesnov ${ }^{1}$, Oleksandr Nechay ${ }^{2}$, Oleksandr Maievskyi ${ }^{3}$, \\ Tetyana Falalyeyeva ${ }^{3}$, Nazarii Kobyliak ${ }^{4}$, Olena Tsyryuk ${ }^{3}$, Yurii Penchuk ${ }^{3}$, Dmytro Shapochka ${ }^{1}$ \\ ${ }^{1}$ Laboratory of Pathology CSD Health Care; ${ }^{2}$ Ukrainian Research and Practical Center for Endocrine Surgery; ${ }^{3}$ Institute of \\ Biology and Medicine, Taras Shevchenko National University of Kyiv; ${ }^{4}$ Department of Endocrinology, Bogomolets National \\ Medical University, Kyiv, Ukraine
}

Background: The relationship between Hashimoto thyroiditis (HT) and papillary thyroid carcinoma (PTC) remains uncertain. We assessed the impact of HT on the tumor immune microenvironment (TIME) in PTC.

Methods: Thirty patients with PTC (group 1) and 30 patients with PTC and HT (group 2) were enrolled in this pilot study. The distribution and number of CD8+ lymphocytes, plasma cells (CD138+), regulatory T cells (forkhead box P3 [FOXP3+)], mast cell tryptase $(\mathrm{MCT}+)$, and $\mathrm{M} 2$ macrophages $(\mathrm{CD} 163+)$ were evaluated. To test the hypothesis that HT impacts PTC development via signal transducer and activator of transcription 6 (STAT6) activation and M2 macrophage polarization, we investigated STAT6 expression in tumor and stromal cells. We also evaluated vascular endothelial growth factor (VEGF) expression by lymph node metastasis (LNM) status.

Results: TIME showed significant between-group differences. Group 1 patients demonstrated immune desert or immune-excluded immunophenotypes, while an inflamed phenotype with more CD8+ cells $(P<0.001)$ predominated in group 2. Immune-excluded TIME was associated with the highest LNM rate. In PTC, LNM was associated with more numerous CD163+ cells. Moreover, LNM in group 1 was associated with increased numbers of mast cells peritumorally and FOXP3+ cells intratumorally and peritumorally. Group 2 demonstrated higher STAT6 but not higher VEGF expression in tumor cells. High VEGF expression was associated with LNM regardless of HT status.

Conclusion: Concomitant HT impacted PTC signaling via STAT6 and TIME by increasing the number of CD8+ cells. LNM is associated with increases in CD163+ cells and VEGF expression in PTC, whereas HT affected LNM through different mechanisms.

Keywords: Thyroid cancer, papillary; Hashimoto disease; Metastasis; T-lymphocytes; Macrophages

\section{INTRODUCTION}

Various countries worldwide, including Ukraine, have observed a recent increase in the number of thyroid cancer cases [1]. The data obtained in the UkrAm Project demonstrated that for at least 21 years after the Chernobyl accident, thyroid cancer risks
Received: 30 December 2019, Revised: 28 March 2020, Accepted: 1 April 2020

Corresponding author: Nazarii Kobyliak

Department of Endocrinology, Bogomolets National Medical University,

Pushkinska 22a St, Kyiv 01601, Ukraine

Tel: +380-44-235-6005, Fax: +380-44-235-6005,

E-mail: nazariikobyliak@gmail.com
Copyright $\odot 2020$ Korean Endocrine Society

This is an Open Access article distributed under the terms of the Creative Commons Attribution Non-Commercial License (https://creativecommons.org/ licenses/by-nc/4.0/) which permits unrestricted non-commercial use, distribution, and reproduction in any medium, provided the original work is properly cited. 
remained significant [2].

There are several well-known risk factors for the development and progression of papillary thyroid carcinoma (PTC). In addition to radiation, genetic alterations, and sex, chronic inflammation is involved in malignant transformation of the thyroid $[3,4]$. While a link between cancer and inflammation has been well-established, such a relationship has not yet been confirmed for inflammatory thyroid diseases [5], the most common of which is Hashimoto thyroiditis (HT) [6]. Chronic inflammation contributes to carcinogenesis by producing mitogenic, antiapoptotic, proangiogenic, and lymphangiogenic factors that could explain the association between HT and PTC [7].

However, concomitant HT in cases of PTC is associated with a better prognosis than PTC alone [8,9]. In fact, PTC patients with concomitant HT exhibited less aggressive clinicopathological characteristics, including lower rates of extrathyroidal extension (ETE) and lymph node metastasis (LNM) $[1,10]$. In the investigation of possible mechanisms of association between these conditions, various biomarkers have been identified as potentially being involved in neoplastic transformation from HT to PTC [11]. However, malignant cells are closely related to the tumor immune microenvironment (TIME), which plays a critical role in tumor development and progression by affecting immune surveillance and tumor spread $[12,13]$.

Different approaches for the evaluation of TIME exist $[12,14]$, and several attempts have been made to classify tumor immunophenotype with regard to TIME. As CD8+ cells are the main cells involved in anti-tumor immune control, most classifications of tumor immunophenotype and assessments of immune score are based on the CD8+ cell count $[12,13]$. However, the relationship between concomitant HT and TIME in PTC is still not clearly understood, nor is it clear which immune-mediated mechanisms are involved in the impact of HT on PTC outcomes. One possible mechanism is the involvement of tumorassociated macrophages (TAMs). It has previously been shown that TAMs displaying the M2 phenotype facilitate neoangiogenesis and matrix remodeling. Interestingly, in HT, TAM polarization that leads to the activation of the M2 phenotype can be induced by the overexpression of the cytokines interleukin (IL)-4 and IL-13 [15]. In addition, IL-4 and IL-13 have been shown to exert their effects via the signal transducer and activator of transcription 6 (STAT6) protein [16]; therefore, activation by IL-4 and IL-13 as part of HT pathogenesis and progression could be a promising mechanism [17]. This supports the hypothesis that HT can affect PTC development through IL-4-STAT6 axis modulation of TIME and M2 macrophage polarization, facilitat- ing angiogenesis and tumor progression via the action of vascular endothelial growth factor (VEGF) $[18,19]$.

However, no data yet exist regarding the effect of HT on TIME, STAT6 expression, and VEGF expression in PTC. In this study, we focused on assessing variations in TIME and differences in the numbers of immune cells in PTC according to the coexistence of HT, as well as the relationships of these data with outcomes. In addition, we assessed the role of innate immune cells involved in the regulation of inflammation, repair, angiogenesis, and tumor growth - namely mast cells and macrophages - that are thought to be key TIME cells.

\section{METHODS}

This study was conducted at the Laboratory of Pathology at CSD Health Care Hospital in collaboration with the Ukrainian Research and Practical Center for Endocrine Surgery. The Institutional Review Board and Local Bioethics Committee of this center approved the study design and the use of human samples (Protocol No. 204 from April 12, 2018). All patients enrolled in the study provided written informed consent.

\section{Study design}

A total of 30 patients with histologically-confirmed conventional PTC (group 1) and 30 patients with PTC and concomitant HT (group 2) were enrolled. HT diagnosis was based on clinical and sonographic data and was confirmed by the presence of serum autoantibodies to thyroglobulin and thyroid peroxidase and by histological evaluation of slides. The following criteria for HT diagnosis were used: diffuse lymphocyte infiltration, lymphoid follicles with germinal centers, and Hürthle cell changes.

\section{Inclusion criteria}

All enrolled cases met the following criteria: (1) female sex (to exclude sex-related differences, only female patients were included in the study); (2) patient age of 21 to 54 years at the time of hospital admission; and (3) histologically-confirmed conventional PTC $>1 \mathrm{~cm}$ but $\leq 4 \mathrm{~cm}$ (pT1b-2) with no ETE. The two groups included equivalent numbers of patients (15 of 30 patients in each group) with and without metastasis.

\section{Exclusion criteria}

The exclusion criteria were as follows: (1) male sex; (2) patient age under 21 years or over 55 years; (3) diagnosis with other malignancies prior to thyroidectomy; (4) non-conventional PTC; (5) micro-carcinoma, tumor larger than $4 \mathrm{~cm}$, or the pres- 
ence of ETE; (6) receipt of hormonal replacement therapy prior to diagnostics and surgery; (7) Graves' disease; (8) pregnancy; and (9) diabetes mellitus.

\section{Immunohistochemical analysis}

To analyze the role of immune-mediated links between PTC and $\mathrm{HT}$, the immunohistochemical investigation was conducted to count various types of immune cells. The cells of interest were cytotoxic T cells (CD8; clone C8/144B, Dako, Glostrup, Denmark) as effectors of cell-mediated antitumor immunity, plasma cells (CD138; clone MI15, Dako) as effectors of humoral immunity, regulatory T (Treg) cells (forkhead box P3 [FOXP3]; clone EP340, Cell Marque, Rocklin, CA, USA) as cells involved in self-tolerance and immunosuppression, mast cell tryptase (MCT; clone AA1, Diagnostic BioSystems, Pleasanton, CA, USA), and tumor-associated M2 macrophages (CD163; clone MRQ-26, Cell Marque) (Supplemental Table S1).

The numbers and distributions of these immune cells were examined. In the evaluation of the cell distribution, we considered the interior of the tumor as well as the peritumoral area. In addition, we counted immune cells in the intact thyroid tissue that was unaffected by PTC. The number of immunopositive cells was assessed per square millimeter. A blinded histomorphometric analysis was performed by two independent observers.

For interpretation of the type of TIME, the following types were considered according to Chen [20]: (1) the immune desert (ID) type, characterized by a lack of pre-existing immunity and a low number of lymphocytes in and around the tumor; (2) the immune-excluded (IE) type, which exhibits prominent peritumor infiltration but a low number of lymphocytes inside the tumor, reflecting the inability of lymphocytes to infiltrate the tumor stroma; and (3) the inflamed type (Inf), in which high infiltration by lymphocytes demonstrates that antitumor $\mathrm{T}$ cells have infiltrated the tumor, but fail to function properly.

To assign TIME type, a semiquantitative evaluation of CD8+ cell infiltration was performed. A threshold of 60 cells per square millimeter was used to stratify CD8+ cell infiltration as either high or low, both in and around the tumor. The ID type was assigned in cases with low CD8+ infiltration in both compartments. The IE type included tumors with low CD8+ infiltration inside the PTC, but high infiltration in the peritumoral area. Finally, cases with high CD8+ infiltration both inside and around PTC foci were considered to be of the Inf immunophenotype. The relationship between immunophenotype and LNM was assessed in both groups.

To test the hypothesis that the impact of HT on PTC is due to
STAT6 activation, we assessed the expression of STAT6 (clone EP325, Cell Marque). In addition, the expression of VEGF (clone VG1, Thermo Fisher Scientific, Waltham, MA, USA) as a key facilitator of tumor angiogenesis and progression was evaluated. STAT6 and VEGF expression levels were assessed in both stroma and tumor cells. The number of immunopositive stromal cells was calculated per square millimeter. The expression levels of STAT6 and VEGF in tumor cells were semi-quantitatively scored on the basis of intensity using a scale of 0 to 3 ( 0 , negative; 1 , weak; 2 , moderate; 3 , strong) and the percentage of positive cells ( $0 \%$, negative; $1 \%$ to $25 \%, 1+; 26 \%$ to $50 \%, 2+$; $>50 \%, 3+)$. The output included a total score, which was derived by multiplying the staining extent by the staining intensity.

\section{Statistical analysis}

Mathematical and statistical processing of the results was carried out using MedCalc software (MedCalc Software, Mariakerke, Belgium). The data were evaluated for the existence of a normal distribution using the Shapiro-Wilk test. Further analysis was performed using one-way analysis of variance. The results were presented in the form of mean \pm standard error of the mean with $95 \%$ confidence interval $(\mathrm{CI})$. $P$ values $\leq 0.05$ were considered to indicate statistical significance.

\section{RESULTS}

Patient age was similar in groups 1 and $2(47.6 \pm 0.65$ years vs. $48.5 \pm 0.48$ years, respectively). PTC size was also comparable, at $18.1 \pm 0.49 \mathrm{~mm}$ (range, 17.1 to 19.0 ) and $19.2 \pm 0.72 \mathrm{~mm}$ (range, 17.8 to 20.7 ), respectively.

\section{Characteristics of TIME in patients with PTC with regard to HT status}

We first assessed the impact of HT on TIME by evaluating the number of immune cells in PTC and PTC+HT patients in the intact thyroid, the peritumoral area, and inside the PTC (in tumor nests in the central part of the tumor). Assessment of PTC histopathology in patients with coexisting HT revealed high infiltration in both intact thyroid tissues and the PTC. In addition, tumor nodules were surrounded with lymphohistiocytic infiltration. Dense lymphocytic infiltration due to HT was associated with changes in thyroid structure, with alteration of the histological architecture and cytological atypia. As expected, the numbers of all immune cells in intact thyroid tissues were significantly higher in patients with PTC and HT than in patients with PTC alone (Table 1). 
PTC areas demonstrated high numbers of M2 macrophages and mast cells regardless of HT coexistence, although their number and distribution varied widely between groups. In contrast, the number of cells involved in adaptive immunity (plasma cells and cytotoxic $\mathrm{T}$ cells) was much higher in group 2 than in group 1, both inside and around the tumor $(P<0.001)$ (Fig. 1).
Interestingly, despite higher numbers of Treg cells in group 2 patients, no statistically significant differences were found in the number of FOXP3 + cells either inside or around the PTC. It is important to note that FOXP3 cells were rare (up to only 5 to 10 cells per square millimeter), suggesting that the lack of Tregmediated immunosuppression could explain the relatively be-

\begin{tabular}{|c|c|c|c|}
\hline \multirow{2}{*}{ Cell no. by compartment } & \multicolumn{2}{|c|}{ Groups of patients } & \multirow{2}{*}{$P$ value } \\
\hline & Group 1 (PTC only) & Group 2 (PTC+HT) & \\
\hline \multicolumn{4}{|l|}{ M2 macrophages (CD163+) } \\
\hline Intact thyroid & $10.1 \pm 0.75(8.61-11.60)$ & $18.1 \pm 2.79(12.58-23.68)$ & 0.002 \\
\hline Peritumoral area & $228.1 \pm 44.4(139.89-316.28)$ & $165.3 \pm 10.3(144.6-185.9)$ & 0.268 \\
\hline Intratumoral area & $180.3 \pm 11.9(156.78-203.90)$ & $194.2 \pm 7.43(179.45-208.96)$ & 0.365 \\
\hline \multicolumn{4}{|l|}{ Mast cells $(\mathrm{MCT}+)$} \\
\hline Intact thyroid & $3.50 \pm 0.45(2.61-4.39)$ & $4.41 \pm 0.71(3.01-5.81)$ & 0.255 \\
\hline Peritumoral area & $54.2 \pm 8.80(36.72-71.66)$ & $37.5 \pm 3.38(30.69-44.31)$ & 0.143 \\
\hline Intratumoral area & $38.0 \pm 3.56(30.97-45.07)$ & $34.4 \pm 2.32(29.79-39.02)$ & 0.447 \\
\hline \multicolumn{4}{|l|}{ Plasma cells (CD138+) } \\
\hline Intact thyroid & $2.59 \pm 0.21(2.17-3.01)$ & $6 \pm 0.66(4.65-7.34)$ & $<0.001$ \\
\hline Peritumoral area & $28.02 \pm 3.11(21.78-34.25)$ & $49.9 \pm 8.81(31.74-68.09)$ & 0.005 \\
\hline Intratumoral area & $11.7 \pm 1.72(8.31-15.18)$ & $66.3 \pm 8.72(48.64-84.01)$ & $<0.001$ \\
\hline \multicolumn{4}{|l|}{ Cytotoxic T cells (CD8+) } \\
\hline Intact thyroid & $8.04 \pm 0.67(6.71-9.36)$ & $77.5 \pm 9.60(58.19-96.86)$ & $<0.001$ \\
\hline Peritumoral area & $52.1 \pm 4.43(43.31-60.89)$ & $201.6 \pm 23.2(155.03-248.30)$ & $<0.001$ \\
\hline Intratumoral area & $27.6 \pm 1.89(23.88-31.39)$ & $184.5 \pm 18.3(147.76-221.27)$ & $<0.001$ \\
\hline \multicolumn{4}{|c|}{ Regulatory T cells (FOXP3+) } \\
\hline Intact thyroid & $0.88 \pm 0.15(0.59-1.18)$ & $2.37 \pm 0.37(1.61-3.12)$ & $<0.001$ \\
\hline Peritumoral area & $2.44 \pm 0.39(1.67-3.21)$ & $1.39 \pm 0.26(0.86-1.93)$ & 0.091 \\
\hline Intratumoral area & $3.78 \pm 0.48(2.84-4.73)$ & $3.73 \pm 0.65(2.42-5.05)$ & 0.955 \\
\hline
\end{tabular}

Values are expressed as mean \pm standard error of the mean ( $95 \%$ confidence interval).

PTC, papillary thyroid carcinoma; HT, Hashimoto thyroiditis; MCT, mast cell tryptase; FOXP3, forkhead box P3.

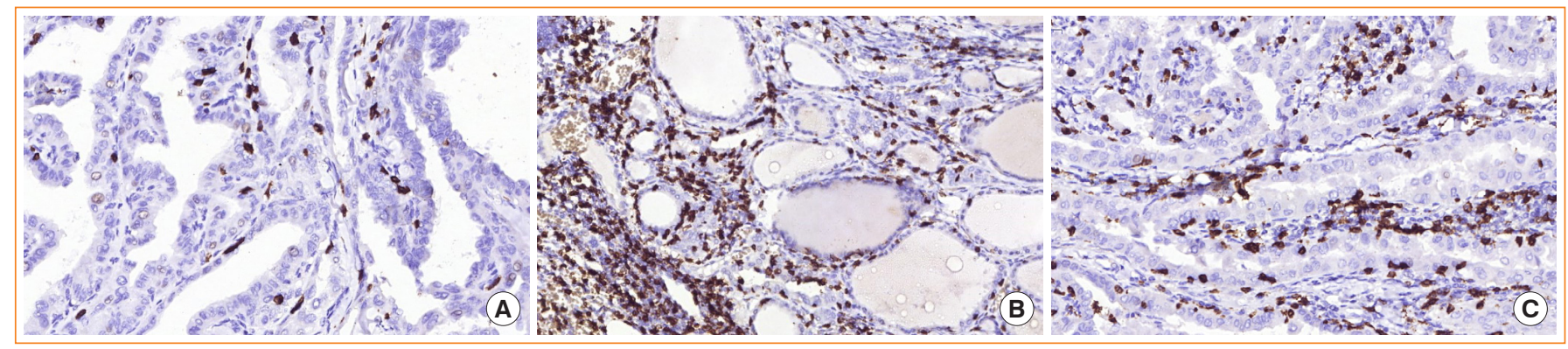

Fig. 1. Differences in the number of cytotoxic T lymphocytes in patients of group 1 (A) and group 2 (B, C). (B) Zone of infiltration of intact thyroid tissue in a patient with concomitant Hashimoto thyroiditis. (C) Intratumoral area. Immunohistochemistry using antibodies to CD8 $(\times 200)$. 
nign progression and outcome of PTC.

Thus, as expected, coexistence with HT was associated with

Table 2. Distribution of Immune Phenotypes by Group of Patients

\begin{tabular}{lccc}
\hline \multirow{2}{*}{ TIME phenotype } & \multicolumn{2}{c}{ Group of patients } & \\
\cline { 2 - 3 } & $\begin{array}{c}\text { Group 1 } \\
\text { (PTC only) }\end{array}$ & $\begin{array}{c}\text { Group 2 } \\
\text { (PTC+HT) }\end{array}$ & \\
\hline ID in total & $16(53.3)$ & 0 & $16(26.7)$ \\
LNM & $6(37.5)$ & 0 & $6(37.5)$ \\
No LNM & $10(62.5)$ & 0 & $10(62.5)$ \\
IE in total & $13(43.4)$ & $3(10)$ & $16(26.7)$ \\
LNM & $9(69.2)$ & $3(100)$ & $12(75)$ \\
No LNM & $4(30.8)$ & 0 & $4(25)$ \\
Inf in total & $1(0.3)$ & $27(90)$ & $28(46.6)$ \\
LNM & 0 & $12(44.4)$ & $12(42.8)$ \\
No LNM & $1(100)$ & $15(55.6)$ & $16(57.2)$ \\
Total number & 30 & 30 & 60
\end{tabular}

Values are expressed as number (\%).

TIME, tumor immune microenvironment; PTC, papillary thyroid carcinoma; HT, Hashimoto thyroiditis; ID, immune desert immunophenotype; LNM, lymph node metastasis; IE, immune-exclusion immunophenotype; Inf, inflamed immunophenotype. changes in tumor immune infiltration characteristics and TIME type (Table 2). The TIME phenotype distribution differed to a significant extent between groups $(P<0.001)$. Group 1 patients demonstrated ID or IE immunophenotypes, while the Inf immunophenotype predominated in group 2.

\section{TIME and the risk of lymph node metastasis}

We next compared the rate of LNM in patients with different TIME types. We found that TIME type was associated with differences in the LNM rate with some group-specific peculiarities (Table 2).

The ID immunophenotype, characterized by a low number of lymphocytes, was found only among group 1 patients, specifically in 16 cases (53.3\% of group 1). LNM was observed in $37.5 \%$ of these patients. The IE type, with prominent peritumor infiltration, was found in 16 patients, 13 of whom were from group $1(81.25 \%)$. Only three cases were in patients with concomitant HT, and all of these cases exhibited LNM. Interestingly, this TIME type was associated with the highest rate of LNM (75\% overall). The Inf immunophenotype, with high lymphocytic infiltration, was found in only one case in group 1 with no LNM. However, it was the predominant TIME type in group 2 (27 of 30 patients; $90 \%$ ), and it was accompanied by LNM in

Table 3. Number of Various Types of Immune Cells in and Around Papillary Thyroid Carcinoma by Immunophenotype

\begin{tabular}{|c|c|c|c|c|}
\hline \multirow{2}{*}{ Immune cells by compartment } & \multicolumn{3}{|c|}{ TIME phenotype } & \multirow{2}{*}{$P$ value } \\
\hline & ID & IE & Inf & \\
\hline \multicolumn{5}{|l|}{ M2 macrophages (CD163+ cells) } \\
\hline Central tumor area & $124.5 \pm 11.5(101.6-147.5)$ & $243 \pm 46.0(46.6-339.4)$ & $222.6 \pm 15.7(190.7-254.6)$ & $<0.001$ \\
\hline Peritumoral area & $71.2 \pm 11.5(48.19-94.23)$ & $262.4 \pm 17.3(227.5-297.3)$ & $153 \pm 20.8(107.6-198.4)$ & $<0.001$ \\
\hline \multicolumn{5}{|l|}{ Mast cells (MCT+ cells) } \\
\hline Central tumor area & $26.9 \pm 3.24(20.5-33.4)$ & $48.8 \pm 6.63(35.5-62.2)$ & $34.5 \pm 3.43(27.6-41.5)$ & \\
\hline Peritumoral area & $12 \pm 2.36(7.24-16.8)$ & $102.9 \pm 16.6(68.6-137.2)$ & $28.9 \pm 5.63(17.1-40.7)$ & $<0.001$ \\
\hline \multicolumn{5}{|l|}{ Cytotoxic T cells (CD8+ cells) } \\
\hline Central tumor area & $25.7 \pm 1.81(22.2-29.4)$ & $43.4 \pm 5.87(31.6-55.3)$ & $249.6 \pm 25.8(197.2-302.1)$ & $<0.001$ \\
\hline Peritumoral area & $21.5 \pm 1.83(17.8-25.2)$ & $116.0 \pm 11.7(92.3-139.7)$ & $214.9 \pm 20.8(172.6-257.3)$ & $<0.001$ \\
\hline \multicolumn{5}{|l|}{ Plasma cells (CD138+ cells) } \\
\hline Central tumor area & $1 \pm 0.43(0.06-1.94)$ & $33.7 \pm 3.94(25.6-41.8)$ & $47.3 \pm 9.17(28.4-66.3)$ & $<0.001$ \\
\hline Peritumoral area & $10.4 \pm 4.36(1.08-19.6)$ & $15.5 \pm 2.56(10.3-20.7)$ & $55.7 \pm 8.25(38.9-72.6)$ & $<0.001$ \\
\hline \multicolumn{5}{|c|}{ Regulatory T cells (FOXP3+ cells) } \\
\hline Central tumor area & $1.39 \pm 0.29(0.79-1.98)$ & $3.74 \pm 0.77(2.17-5.32)$ & $1.17 \pm 0.64(0.17-2.53)$ & $<0.001$ \\
\hline Peritumoral area & $2.37 \pm 0.49(1.39-3.35)$ & $5.91 \pm 0.80(4.28-7.55)$ & $1.76 \pm 0.56(0.57-2.95)$ & $<0.001$ \\
\hline
\end{tabular}




\section{$44.4 \%$ of cases.}

In addition to the numbers of CD8+ cells, we assessed whether the TIME type was related to variability in the abundance of other immune cell types. To do so, we evaluated the numbers of various cells in patients with different TIME types (Table 3). PTC with the IE immunophenotype was associated with the highest infiltration by M2 macrophages, mast cells, and Treg cells $(P<0.001)$, which could explain the relatively high rate of LNM in this group.

Thus, the TIME type was associated with diversity in the quantity and spatial distribution of immune cells, as well as with the risk of LNM.

\section{Cellular mechanisms of LNM in patients with and without HT}

To determine which cells play key roles in LNM development, we evaluated the numbers of different cells with regard to the coexistence of HT and LNM. As shown in Fig. 2, LNM development in patients with PTC alone was associated with an increased number of M2 macrophages in both the central tumor area and the peritumor zone. No statistically significant difference in MCT number was observed between group 1 patients with and without LNM (Fig. 3). Surprisingly, LNM was associated with slightly increased infiltration of the peritumoral area with $\mathrm{CD} 8+(P=0.024)$ and $\mathrm{CD} 138+(P=0.008)$ cells, reflecting the role of the IE TIME type. In addition, LNM in PTC with HT was accompanied by an elevated number of FOXP3 cells in both the central and peritumoral areas $(P<0.001)$.

In contrast, patients with concomitant HT exhibited the involvement of different cells. Progression of PTC in group 2 patients was associated with a significant increase in the number of mast cells, a slight elevation of the number of M2 macrophages, and a prominent decrease in CD8+ and CD138+ cells in both the central tumor and the peritumoral area. However, we

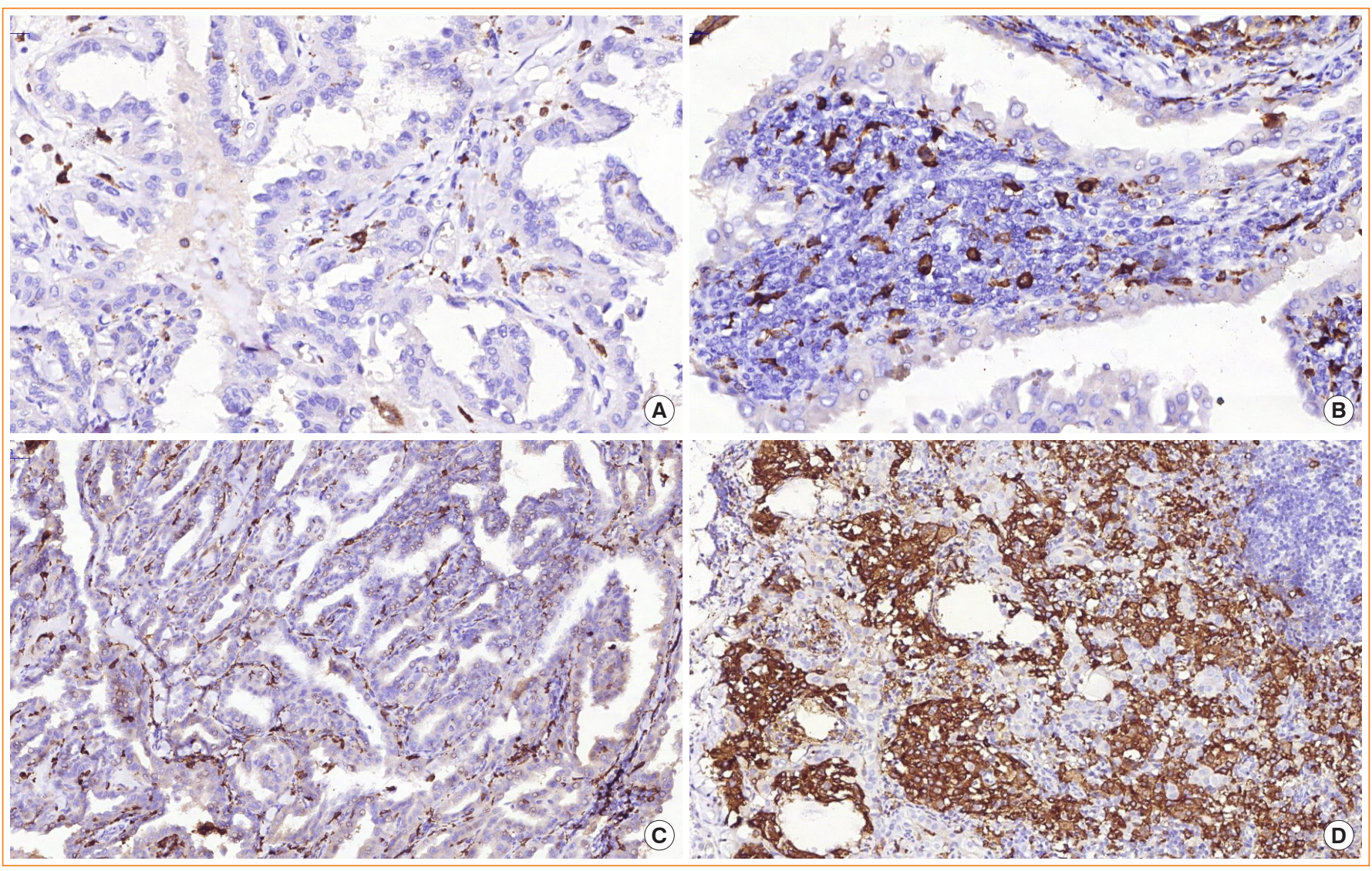

Fig. 2. An increased number of M2 macrophages was independently associated with lymph node metastasis (LNM) development in patients with concomitant Hashimoto thyroiditis. (A) A patient in group 1 without LNM demonstrated a low number of M2 macrophages $(\times 200)$. (B) A patient in group 2 without LNM $(\times 200)$. (C) A high number of M2 macrophages in papillary thyroid carcinoma with LNM $(\times 100)$. (D) Numerous M2 macrophages around metastases of thyroid cancer in the lymph node $(\times 100)$. Immunohistochemistry using antibodies to CD163 (×200). 

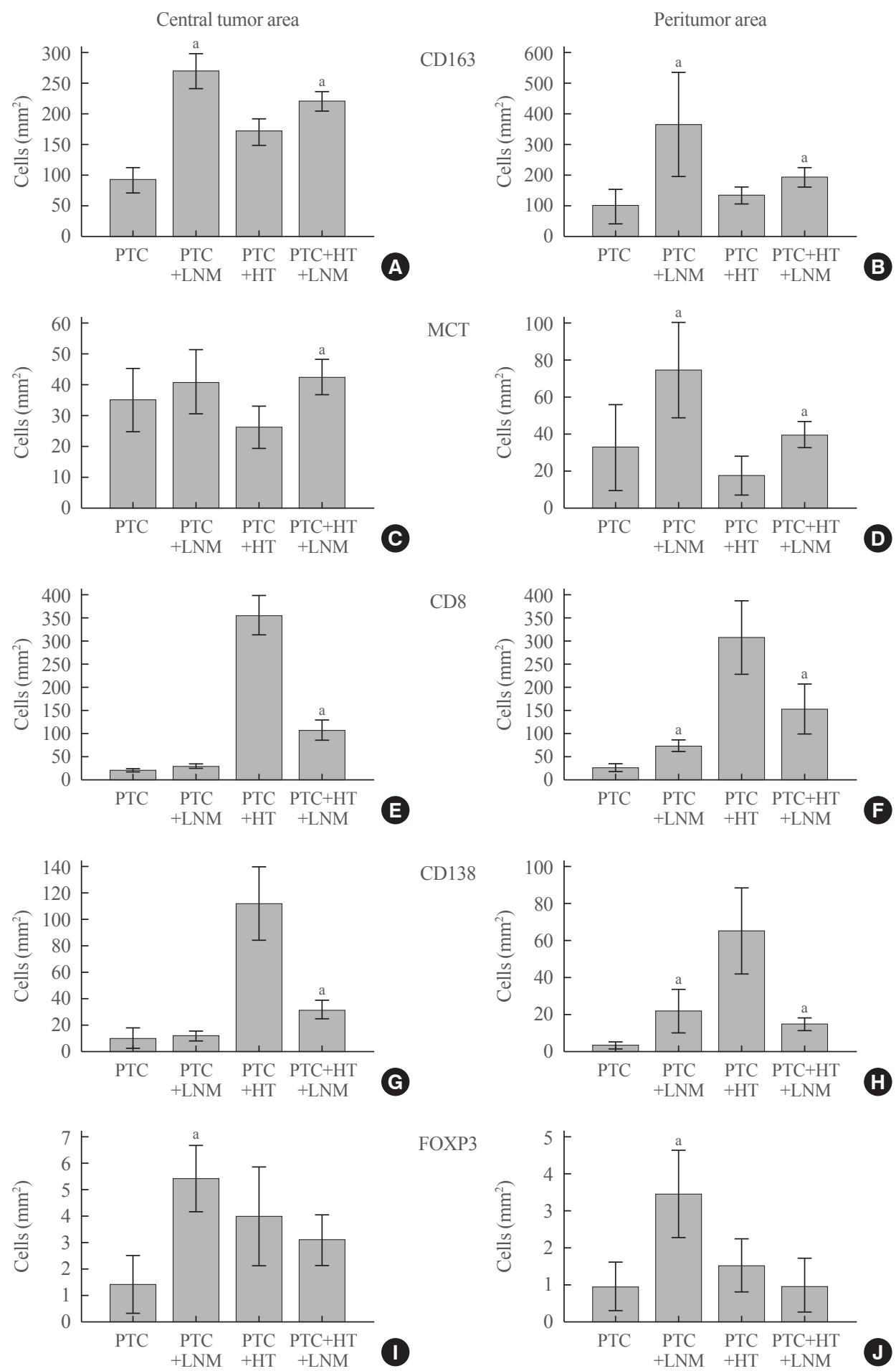

Fig. 3. The number of immune cells in and around papillary thyroid carcinoma (PTC) in patients with and without concomitant Hashimoto thyroiditis (HT) by lymph node metastasis (LNM) status. (A, B) Number of M2 macrophages (CD163 positive cells) within tumor and in peritumor stroma respectively. (C, D) Count of mast cells (mast cell tryptase [MCT] positive cells) within tumor and in peritumor stroma respectively. (E, F) Number of T-cytotoxic lymphocytes (CD8 positive cells) within tumor and in peritumor stroma respectively. (G, H) Count of plasma cells (CD138 positive cells) within tumor and in peritumor stroma respectively. (I, J) Number of T-regulatory cells (forkhead box

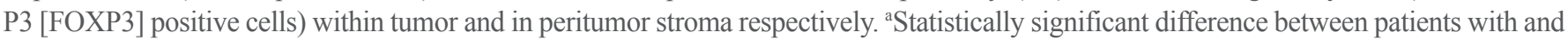
without LNM at $P<0.05$. 
found no significant differences in Treg number regarding LNM status.

Thus, PTC with concomitant HT demonstrated a specific immune profile, and different mechanisms of PTC progression were found depending on HT status.

\section{STAT6 and VEGF expression in patients with PTC with regard to $H$ T coexistence and LNM}

To discover possible mechanisms behind the relationship between immune-mediated inflammation and PTC characteristics, we assessed the expression of STAT6 and VEGF. Surprisingly, in addition to a stromal immunopositive reaction to STAT6, we observed STAT6 expression in tumor cells inside the PTC. Stromal cells had strong immunoreactivity $(3+)$ and widely varied in number, correlating with the count of M2 macrophages ( $r=$ $0.648, P<0.001)$. This supports the role of STAT6 in macrophage polarization.

While intact follicular cells did not express STAT6, tumor cells inside the PTC demonstrated mild to high expression of STAT6, the total score of which correlated with the degree of lymphocytic infiltration $(r=0.816, P<0.001)$ and which was significantly higher in group 2 patients $(P=0.001)$ (Fig. 4). In addition, in group 2 patients, thyroid tissue unaffected by PTC demonstrated a STAT6-positive reaction that was associated with histological and cytological atypia of follicular cells. This reflects the direct immune-mediated mechanisms of STAT6 activation in follicular tumor cells and indicates a need for further investigation of its role in tumor transformation and growth in patients with HT. However, we found no differences in STAT6 expression in PTC cells with regard to LNM status.

VEGF expression was also observed both in stromal cells and inside PTC cells. Numerous VEGF-positive stromal cells were present and corresponded in number and distribution to CD163+ cells. In addition, mild to moderate VEGF expression was observed in the tumor cells of the PTC. The total score for VEGF expression in tumor cells varied from 2 to 6 . We found no difference in the total VEGF expression score in PTC cells between patients with and without coexisting HT. In addition, no significant correlation was observed between VEGF expression and STAT6 expression. However, the intensity of VEGF expression in PTC cells was significantly higher in patients with LNM independent of HT coexistence (Fig. 4).

Thus, assessment of STAT6 and VEGF expression in patients of varying node status showed that the expression of STAT6 was slightly lower in patients with metastasis; however, this difference was not statistically significant. In contrast, high VEGF expression was associated with LNM $(P<0.001)$, reflecting the relationship between this growth factor and PTC progression.

\section{DISCUSSION}

A key factor in the determination of tumor progression is the proportion and spatial distribution of cytotoxic $\mathrm{T}$ lymphocytes within the TIME. It has been reported that de novo carcinogenesis is associated with the inability of antitumor T cells to control tumor growth due to tumor-induced tolerance mechanisms [21].

The results of the present study revealed that the TIME in PTC had varying characteristics that were associated with the presence or absence of concomitant HT. HT coexistence was found to be associated with an elevated number of CD8+ cells; this supported the results of previous studies that showed that HT promotes the recruitment of $\mathrm{T}$ lymphocytes, including CD4+ cells, cytotoxic T lymphocytes (CD8+), and Th17 cells, to the thyroid gland [22]. In addition, HT patients exhibited significantly higher expression of the Th1 transcription factor T-bet than did healthy controls [23]. Th1 cells are induced in response to interferon- $\gamma$ and IL-12, which are secreted primarily by dendritic and natural killer cells and which promote cytotoxic T cell proliferation and differentiation [24]. This could explain the higher number of CD8+ cells in PTC with HT than in PTC alone. In patients with concomitant HT, the number of CD8+ cells was significantly higher in the PTC interior than in intact thyroid tissue, affecting the TIME. To the extent that the number of CD8+ cells reflects the activation of the antitumor response, this finding supports the concept that HT coexistence could facilitate the activation of antitumor immunity and the promotion of a cancer immune cycle that affects TIME characteristics.

Currently, three broad classes of TIME have been described on the basis of heterogeneity in the spatial distribution of CD8+ lymphocytes [12]. Tumors with the ID immunophenotype are characterized by an absence of CD8+ cells that corresponds to a lack of pre-existing antitumor immunity. This phenotype can reflect either poor tumor immunogenicity due to a low mutational load or the lack of antigen release or failure of antigen presentation and T-cell activation due to insufficiency of dendritic cells or disrupted costimulatory interactions [20].

The IE phenotype is associated with an abundance of CD8+ cells in the tumor stroma in the peritumoral area. However, the $\mathrm{T}$ cells cannot reach tumor cells, which reflects altered mechanisms of T-lymphocyte recruitment into tumor nests due to a lack of chemokines or low levels of chemokine receptors [25]. In addition, high expression of molecules responsible for vascu- 

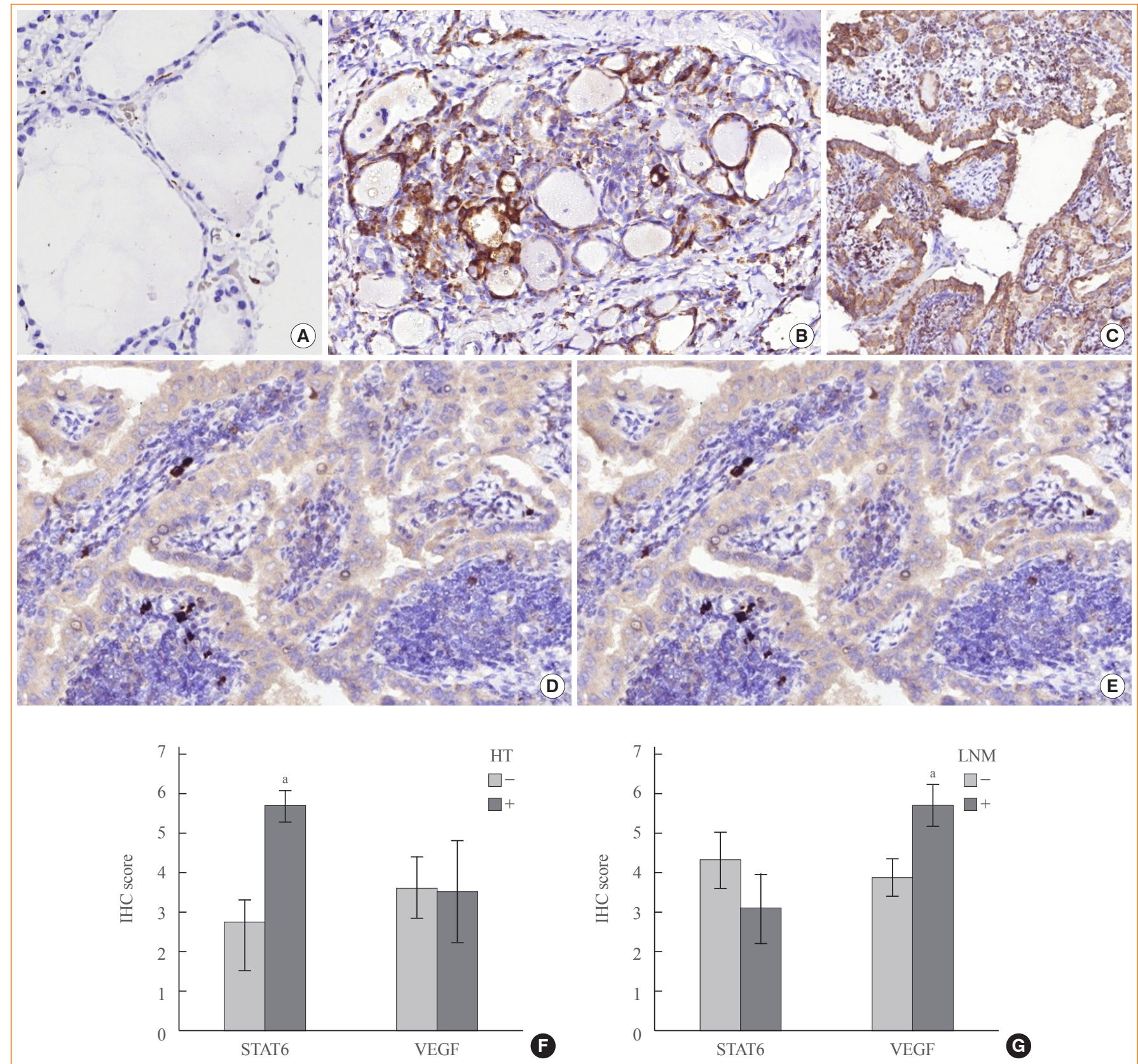

Fig. 4. Signal transducer and activator of transcription 6 (STAT6) and vascular endothelial growth factor (VEGF) expression in patients with papillary thyroid carcinoma (PTC) according to Hashimoto thyroiditis (HT) and lymph node metastasis (LNM). (A) STAT6 expression in patients of group 2 in the intact thyroid $(\times 200)$. (B) STAT6 expression in follicular cells in areas of lymphocyte infiltration associated with histoarchitectural and cytological atypia in group $2(\times 200)$. (C) STAT6 expression in group 2 patients $(\times 100)$. (D) Low VEGF expression in group 2 patients without LNM. (E) High expression of VEGF in PTC tumor cells associated with LNM. Immunohistochemistry $(\times 200)$. $(\mathrm{F}$, G) Relationship between STAT6 and VEGF expression in the tumor cells of patients with PTC according to HT (F) and LNM (G) status. IHC, immunohistochemical. aStatistically significant difference in the total score of biomarker expression regarding HT or LNM status at $P<0.05$.

logenesis and angiogenesis, such as VEGF, can render antitumor immunity ineffective [19].

Interestingly, most PTC cases in group 1 were associated with the ID or IE immunophenotype. This reflects the alteration of early steps of the cancer immune cycle, including either antigen recognition and presentation with activation of $\mathrm{T}$ cells or $\mathrm{CD} 8+$ 
cell trafficking into the tumor [26]. In contrast, PTC with HT demonstrated predominantly an Inf immunophenotype, which is characterized by an abundance of tumor-infiltrating immune cells, including CD8+ T cells. In addition, HT coexistence with the Inf immunophenotype was associated with a relatively high number of plasma cells, reflecting activation of both cell-mediated and humoral immunity.

The Inf immunophenotype is typical for immunologically hot tumors; however, the abundance of tumor-infiltrating lymphocytes is associated with the arrest of antitumor immunity [27]. This may be related to various factors, including a lack of the major histocompatibility complex on tumor cells, high expression of immune checkpoint markers, or the effects of immunosuppressive cells such as Treg cells and myeloid-derived suppressor cells [28].

In an attempt to decipher the mechanisms of immunosuppression, we assessed the number and spatial distribution of Treg cells. Under physiological conditions, Treg cells play essential roles in maintaining self-tolerance and protecting against autoimmunity [29]. Accordingly, Treg cell depletion has been shown to promote autoimmune thyroiditis [22]. However, Treg cells can suppress antitumor immunity, thereby hindering immunosurveillance against cancer development and progression.

Previous studies have shown that elevation of Treg cells in primary tumors is associated with metastasis and poor prognosis [30]. These cells facilitate tumor immune escape by producing cytokines (IL-10, transforming growth factor- $\beta$, and IL-35) and by inhibiting T-cell receptor activation [31]. However, in our study, we found that most patients with PTC had few Treg cells. Although Treg cells were more numerous in cases of PTC with coexisting HT, the overall number of these cells was low and did not significantly differ between intact and tumor-affected tissues. Only a few patients had high numbers of FOXP3, and all of those patients exhibited IE and LNM.

Thus, concomitant HT impacts the TIME by increasing the number of $\mathrm{CD} 8+$ cells inside and around the tumor, reflecting the facilitation of antitumor immunity. Nonetheless, in the future, the roles of other cells in PTC progression should be assessed in some cases involving LNM.

Another component of TIME that greatly impacts tumor growth and the promotion of immune evasion involves tumorinfiltrating macrophages [27]. It is widely accepted that hypoxia and metabolic reprogramming can promote the polarization of TAMs from the pro-inflammatory M1 type to the immunosuppressive M2 phenotype [32]. Classically activated M1-like macrophages are able to kill tumor cells directly through an ear- ly elimination phase of immuno-editing orchestrated by CD8+ cytotoxic T lymphocytes and interferons [33]. However, M2 macrophages are much more abundant in malignancies [31]. The presence of these cells correlates with angiogenesis, immunosuppression, and the promotion of cancer growth and metastasis [34].

We found no significant difference in the number of CD163+ M2 macrophages between patients with only PTC and those with PTC and concomitant HT. However, a comparison of the number of CD163+ cells in PTC of different immunophenotypes revealed that the IE type was associated with the highest number of M2 cells in both the central tumor area and the peritumoral stroma. In addition, significant changes were found in the number of mast cells in the peritumoral area in PTC with the IE immunophenotype. These findings align with the common concept of the role of M2 macrophages in immunosuppression, which is realized through diverse mechanisms. First, M2 cells can express programmed death ligand 1 (PD-L1) and induce PD-L1 expression on dendritic cells [35]. PD-L1, as well as programmed death ligand 2, bind programmed cell death protein 1 on T cells, suppressing their functions. Second, CD163+ macrophages secrete immunosuppressive cytokines such as IL10 and transforming growth factor- $\beta$, which directly suppress CD8+ T cells and dendritic cells [33]. Moreover, due to the expression of arginase and indoleamine, 2,3-dioxygenase M2 cells are responsible for metabolic reprogramming and starvation in T cells [34].

As the IE immunophenotype of PTC was associated with the highest rate of LNM among the observed cases, it seems that an increased number of M2 macrophages and mast cells inside the tumor, and especially in peritumor compartments, and their prevalence along with $\mathrm{CD} 8+$ cells are associated with tumor progression. To analyze the role of various immune cells in PTC progression, we compared the number of immune cells in PTC with and without metastases and with and without concomitant HT.

Depending on HT status, PTC demonstrates different mechanisms of antitumor immunity failure, as reflected by the tumor immunophenotype. As expected, LNM development in patients with PTC alone was associated with an increased number of M2 cells in both the primary tumor and lymph nodes affected by metastases, confirming the role of CD163 cells in PTC progression. In addition, LNM development in group 1 patients was associated with a high number of mast cells in the peritumoral area and an increase in the number of FOXP3+ cells inside and around the PTC. The increased number and role of mast cells in 
thyroid cancer has been shown previously [36]. These cells are responsible for the production of numerous proangiogenic factors, including VEGFs [25]. Accordingly, in the present study, PTC with LNM was associated with increased expression of VEGF. This proangiogenic growth factor was expressed in both stromal and tumor cells. Several types of stromal cells are involved in VEGF expression, including M2 macrophages and mast cells, and these contribute to neoangiogenesis [37]. However, tumor cell expression of VEGF was associated with PTC aggressiveness and LNM development.

Interestingly, LNM development in PTC with concomitant HT was associated with other immune-mediated mechanisms. In group 2, metastasis was associated with decreased numbers of CD8+ and CD138+ cells and increased numbers of CD163 and mast cells in both central and peritumoral areas, although the difference in M2 macrophage number was less prominent than in group 1. In addition, in group 2, no significant difference was found regarding FOXP3 cell number in patients with and without LNM.

Such differences demonstrate that LNM development in patients with coexisting HT is associated with the failure of cytotoxic T cells. However, regardless of HT status, PTC progression was associated with an increased number of M2 macrophages.

This fact underscores the importance of understanding the molecular control of macrophage phenotype and the mechanisms of TAM polarization. As previously mentioned, IL-4 and IL-13 are involved in M2-phenotype polarization via STAT6, which mediates the transcriptional activation of M2 macrophage-specific genes such as arginase 1 (Arg1), mannose receptor $1(M r c 1)$, resistin-like alpha (Retnla, Fizz1), chitinase-like protein 3 (Chil3, Yml), and the chemokine genes $\mathrm{Ccll} 7$ and Ccl24 [38].

In this study, we assessed the expression of STAT6 and its relationship with tumor characteristics. STAT6 expression was observed in both tumor cells and the stroma. Stromal cells displayed a high-intensity immunopositive reaction that was correlated with the number of M2 macrophages. However, tumor cells expressed STAT6 in different patterns. The highest level of STAT6 expression was found in a patient with PTC and concomitant HT, and this expression correlated with the density of lymphocytic infiltration. In addition, we observed STAT6 expression in atypical thyroid cells outside of the PTC in zones with dense infiltration by lymphocytes. The STAT6 signaling pathway is believed to induce epigenetic changes, leading to decreased responsiveness to inflammatory signals [39]; in this fashion, immune-mediated STAT6 activation in follicular and PTC cells may be a form of adaptation to immune attack [18]. In contrast, STAT6 mediates antiapoptotic biological responses through the induction of BCL2L1/BCL-XL, which could be a mechanism to evade the apoptosis of tumor cells [17]. Therefore, STAT6 is involved not only in macrophage polarization, but also in tumor cell transformation and biology under conditions of chronic inflammation. Nevertheless, we found no significant relationship between STAT6 expression in tumor cells and LNM development. Thus, PTC with coexisting HT demonstrates a specific immune profile, and the mechanisms of PTC progression differ depending on HT status.

In this pilot study, we demonstrated that HT coexistence was associated with higher numbers of CD8+ and CD138+ cells in the PTC and peritumoral area. Regarding TIME, HT coexistence was associated with predominance of the Inf immunophenotype, whereas PTC without HT predominantly demonstrated the ID or IE immunophenotype. The IE immunophenotype was associated with a higher rate of LNM due to prominent M2 macrophages and mast cell infiltration. HT coexistence was associated with STAT6 expression in tumor cells, but did not affect VEGF expression in either stromal cells or PTC cells. The upregulation of VEGF in PTC cells was associated with LNM development independently of HT coexistence.

The main limitation of this study is the small number of cases. In addition, only middle-aged women with conventional T1bT2 PTC were enrolled in the study. The lack of genetic testing may also have affected the results.

\section{CONFLICTS OF INTEREST}

No potential conflict of interest relevant to this article was reported.

\section{AUTHOR CONTRIBUTIONS}

Conception or design: O.S., O.C., O.N., N.K. Acquisition, analysis, or interpretation of data: O.C., O.N., O.T., Y.P., D.S. Drafting the work or revising: O.N., O.M., T.F., D.S. Final approval of the manuscript: O.S., N.K.

\section{ORCID}

Oksana Sulaieva https://orcid.org/0000-0002-9614-4652 Nazarii Kobyliak https://orcid.org/0000-0001-9814-689X 


\section{REFERENCES}

1. Zhu F, Shen YB, Li FQ, Fang Y, Hu L, Wu YJ. The effects of Hashimoto thyroiditis on lymph node metastases in unifocal and multifocal papillary thyroid carcinoma: a retrospective Chinese cohort study. Medicine (Baltimore) 2016; 95:e2674.

2. Tronko M, Mabuchi K, Bogdanova T, Hatch M, Likhtarev I, Bouville A, et al. Thyroid cancer in Ukraine after the Chernobyl accident (in the framework of the Ukraine-US Thyroid Project). J Radiol Prot 2012;32:N65-9.

3. Lee KH, Seok EY, Kim EY, Yun JS, Park YL, Park CH. Different prognostic values of individual hematologic parameters in papillary thyroid cancer due to age-related changes in immunity. Ann Surg Treat Res 2019;96:70-7.

4. Sulaieva O, Chernenko O, Chereshneva Y, Tsomartova D, Larin O. Thyroid stimulating hormone levels and BRAFV600E mutation contribute to pathophysiology of papillary thyroid carcinoma: relation to outcomes? Pathophysiology 2019;26: 129-35.

5. Resende de Paiva C, Gronhoj C, Feldt-Rasmussen U, von Buchwald C. Association between Hashimoto's thyroiditis and thyroid cancer in 64,628 patients. Front Oncol 2017;7:53.

6. Konturek A, Barczynski M, Wierzchowski W, Stopa M, Nowak W. Coexistence of papillary thyroid cancer with Hashimoto thyroiditis. Langenbecks Arch Surg 2013;398: 389-94.

7. Fang W, Ye L, Shen L, Cai J, Huang F, Wei Q, et al. Tumorassociated macrophages promote the metastatic potential of thyroid papillary cancer by releasing CXCL8. Carcinogenesis 2014;35:1780-7.

8. Borowczyk M, Janicki A, Dworacki G, Szczepanek-Parulska E, Danieluk M, Barnett J, et al. Decreased staging of differentiated thyroid cancer in patients with chronic lymphocytic thyroiditis. J Endocrinol Invest 2019;42:45-52.

9. Marotta V, Sciammarella C, Chiofalo MG, Gambardella C, Bellevicine C, Grasso M, et al. Hashimoto's thyroiditis predicts outcome in intrathyroidal papillary thyroid cancer. Endocr Relat Cancer 2017;24:485-93.

10. Huang BY, Hseuh C, Chao TC, Lin KJ, Lin JD. Well-differentiated thyroid carcinoma with concomitant Hashimoto's thyroiditis present with less aggressive clinical stage and low recurrence. Endocr Pathol 2011;22:144-9.

11. Means C, Clayburgh DR, Maloney L, Sauer D, Taylor MH, Shindo ML, et al. Tumor immune microenvironment characteristics of papillary thyroid carcinoma are associated with histopathological aggressiveness and BRAF mutation status. Head Neck 2019;41:2636-46.

12. Binnewies M, Roberts EW, Kersten K, Chan V, Fearon DF, Merad M, et al. Understanding the tumor immune microenvironment (TIME) for effective therapy. Nat Med 2018;24: 541-50.

13. Caruana I, Simula L, Locatelli F, Campello S. T lymphocytes against solid malignancies: winning ways to defeat tumours. Cell Stress 2018;2:200-12.

14. Can NY, Ayturk S, Celik M, Sezer YA, Ozyilmaz F, Tastekin E, et al. Histological perspective on the effects of tumor-associated macrophages in the tumor microenvironment surrounding papillary thyroid carcinoma. Pol J Pathol 2016;67:332-44.

15. Binnemars-Postma K, Bansal R, Storm G, Prakash J. Targeting the Stat6 pathway in tumor-associated macrophages reduces tumor growth and metastatic niche formation in breast cancer. FASEB J 2018;32:969-78.

16. Hebenstreit D, Wirnsberger G, Horejs-Hoeck J, Duschl A. Signaling mechanisms, interaction partners, and target genes of STAT6. Cytokine Growth Factor Rev 2006;17:173-88.

17. Yu T, Gan S, Zhu Q, Dai D, Li N, Wang H, et al. Modulation of M2 macrophage polarization by the crosstalk between Stat6 and Trim24. Nat Commun 2019;10:4353.

18. Czimmerer Z, Daniel B, Horvath A, Ruckerl D, Nagy G, Kiss M, et al. The transcription factor STAT6 mediates direct repression of inflammatory enhancers and limits activation of alternatively polarized macrophages. Immunity 2018;48:75-90.

19. de Araujo-Filho VJ, Alves VA, de Castro IV, Lourenco SV, Cernea CR, Brandao LG, et al. Vascular endothelial growth factor expression in invasive papillary thyroid carcinoma. Thyroid 2009;19:1233-7.

20. Chen DS, Mellman I. Oncology meets immunology: the cancer-immunity cycle. Immunity 2013;39:1-10.

21. Willimsky G, Blankenstein T. Sporadic immunogenic tumours avoid destruction by inducing T-cell tolerance. Nature 2005;437:141-6.

22. Gonzalez-Amaro R, Marazuela M. T regulatory (Treg) and T helper 17 (Th17) lymphocytes in thyroid autoimmunity. Endocrine 2016;52:30-8.

23. Safdari V, Alijani E, Nemati M, Jafarzadeh A. Imbalances in T cell-related transcription factors among patients with Hashimoto's thyroiditis. Sultan Qaboos Univ Med J 2017;17:e17480.

24. Antonelli A, Ferrari SM, Corrado A, Di Domenicantonio A, Fallahi P. Autoimmune thyroid disorders. Autoimmun Rev 
2015; 14:174-80.

25. Galdiero MR, Varricchi G, Marone G. The immune network in thyroid cancer. Oncoimmunology 2016;5:e1168556.

26. Horton BL, Fessenden TB, Spranger S. Tissue site and the cancer immunity cycle. Trends Cancer 2019;5:593-603.

27. Bindea G, Mlecnik B, Tosolini M, Kirilovsky A, Waldner M, Obenauf AC, et al. Spatiotemporal dynamics of intratumoral immune cells reveal the immune landscape in human cancer. Immunity 2013;39:782-95.

28. Xiong H, Mittman S, Rodriguez R, Pacheco-Sanchez P, Moskalenko M, Yang Y, et al. Coexpression of inhibitory receptors enriches for activated and functional $\mathrm{CD} 8^{+} \mathrm{T}$ cells in murine syngeneic tumor models. Cancer Immunol Res 2019; 7:963-76.

29. Spranger S. Mechanisms of tumor escape in the context of the T-cell-inflamed and the non-T-cell-inflamed tumor microenvironment. Int Immunol 2016;28:383-91.

30. Togashi Y, Shitara K, Nishikawa H. Regulatory T cells in cancer immunosuppression: implications for anticancer therapy. Nat Rev Clin Oncol 2019;16:356-71.

31. Liotti F, Prevete N, Vecchio G, Melillo RM. Recent advances in understanding immune phenotypes of thyroid carcinomas: prognostication and emerging therapies. F1000Res 2019;8(F1000 Faculty Rev):227.

32. Condeelis J, Pollard JW. Macrophages: obligate partners for tumor cell migration, invasion, and metastasis. Cell 2006; 124:263-6.

33. Ferrari SM, Fallahi P, Galdiero MR, Ruffilli I, Elia G, Ragusa F, et al. Immune and inflammatory cells in thyroid cancer microenvironment. Int J Mol Sci 2019;20:4413.

34. Quaranta V, Schmid MC. Macrophage-mediated subversion of anti-tumour immunity. Cells 2019;8:747.

35. Loke P, Allison JP. PD-L1 and PD-L2 are differentially regulated by Th1 and Th2 cells. Proc Natl Acad Sci U S A 2003;100:5336-41.

36. Melillo RM, Guarino V, Avilla E, Galdiero MR, Liotti F, Prevete N, et al. Mast cells have a protumorigenic role in human thyroid cancer. Oncogene 2010;29:6203-15.

37. Movahedi K, Laoui D, Gysemans C, Baeten M, Stange G, Van den Bossche J, et al. Different tumor microenvironments contain functionally distinct subsets of macrophages derived from Ly6C(high) monocytes. Cancer Res 2010;70: 5728-39.

38. Gordon S, Martinez FO. Alternative activation of macrophages: mechanism and functions. Immunity 2010;32:593604.

39. Zhang Y, Zhang Y, Gu W, Sun B. TH1/TH2 cell differentiation and molecular signals. Adv Exp Med Biol 2014;841:1544. 